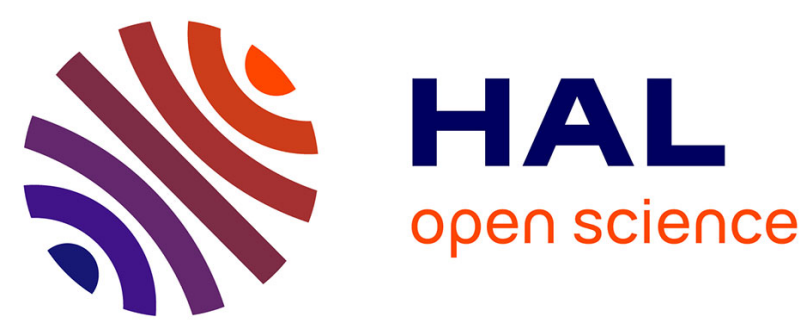

\title{
Short-range wideband FMCW radar for millimetric displacement measurements
}

Andrei Anghel, Gabriel Vasile, Remus Cacoveanu, Cornel Ioana, Silviu

Ciochina

\section{- To cite this version:}

Andrei Anghel, Gabriel Vasile, Remus Cacoveanu, Cornel Ioana, Silviu Ciochina. Short-range wideband FMCW radar for millimetric displacement measurements. IEEE Transactions on Geoscience and Remote Sensing, 2014, 52 (9), pp.5633-5642. 10.1109/TGRS.2013.2291573 . hal-00974718

\section{HAL Id: hal-00974718 \\ https://hal.science/hal-00974718}

Submitted on 7 Apr 2014

HAL is a multi-disciplinary open access archive for the deposit and dissemination of scientific research documents, whether they are published or not. The documents may come from teaching and research institutions in France or abroad, or from public or private research centers.
L'archive ouverte pluridisciplinaire HAL, est destinée au dépôt et à la diffusion de documents scientifiques de niveau recherche, publiés ou non, émanant des établissements d'enseignement et de recherche français ou étrangers, des laboratoires publics ou privés. 


\title{
SHORT-RANGE WIDEBAND FMCW RADAR FOR MILLIMETRIC DISPLACEMENT MEASUREMENTS
}

\author{
Andrei Anghel, Student Member, IEEE, Gabriel Vasile, Member, IEEE, Remus Cacoveanu, Member, IEEE, \\ Cornel Ioana, Member, IEEE, and Silviu Ciochina, Member, IEEE
}

\begin{abstract}
The frequency modulated continuous wave (FMCW) radar is an alternative to the pulse radar when the distance to the target is short. Typical FMCW radar implementations have a homodyne architecture transceiver which limits the performances for short-range applications: the beat frequency can be relatively small and placed in the frequency range affected by the specific homodyne issues (DC offset, self-mixing and 1/f noise). Additionally, one classical problem of a FMCW radar is that the voltage controlled oscillator adds a certain degree of nonlinearity which can cause a dramatic resolution degradation for wideband sweeps. This paper proposes a short-range $X$-band FMCW radar platform which solves these two problems by using a heterodyne transceiver and a wideband nonlinearity correction algorithm based on high-order ambiguity functions and time resampling. The platform's displacement measurement capability was tested on range profiles and synthetic aperture radar (SAR) images acquired for various targets. The displacements were computed from the interferometric phase and the measurement errors were situated below $0.1 \mathrm{~mm}$ for metal bar targets placed at a few meters from the radar.
\end{abstract}

Index Terms-Frequency Modulated Continuous Wave (FMCW), Interferometry, Synthetic Aperture Radar (SAR), Nonlinearity Correction, High-Order Ambiguity Function (HAF), Time Resampling.

\section{INTRODUCTION}

$\mathbf{T}$ HE frequency modulated continuous wave (FMCW) radar is an alternative to the pulse radar when the target range can be relatively small (below $100 \mathrm{~m}$ ). In order to measure such distances with a pulse radar the switching time between transmission and reception should be at most tens of nanoseconds. For a FMCW radar the range information is provided by beat frequencies and each frequency corresponds to a target placed at a certain distance. Typical FMCW radar implementations have a homodyne architecture transceiver [1], [2] which limits the performances for shortrange applications. In the case of targets positioned near the radar, the beat frequency is small and can be situated in

A. Anghel is with the Telecommunications Department, University Politehnica of Bucharest (UPB), 060032 Bucharest, Romania (e-mail: andrei.anghel@munde.pub.ro), and also with the Grenoble Image sPeech Signal Automatics Laboratory (GIPSA-Lab), CNRS / Grenoble INP, 38402 Grenoble, France.

G. Vasile and C. Ioana are with the GIPSA-Lab, CNRS / Grenoble INP, 38402 Grenoble, France (e-mail: gabriel.vasile@gipsa-lab.grenoble-inp.fr; cornel.ioana@gipsa-lab.grenoble-ino.fr).

R. Cacoveanu and S. Ciochina are with the Telecommunications Department, UPB, 060032 Bucharest, Romania (e-mail: cacoveanu@munde.pub.ro; silviu@comm.pub.ro). the frequency band affected by the classical problems of the homodyne architecture (DC offset, self-mixing and 1/f noise) [3]. Another problem of the FMCW transceiver is that the voltage controlled oscillator (VCO) adds a certain degree of nonlinearity which leads to a deteriorated resolution by spreading the target energy through different frequencies [4]. This issue is usually solved either by hardware [5], [6] or software [7], [8] approaches.

This paper presents a short-range FMCW X-band radar based on a heterodyne architecture of the transceiver which eliminates the low frequency self-mixing spectrum [9] and reduces the noise bandwidth. In other works like [10], [11], [12], [13] the heterodyne architecture is used for S-band and $\mathrm{X}$-band FMCW transceivers in order to build a hardware range-gate based on narrow-band communication filters. The range-gate is used either to eliminate from the beat signal some powerful reflections that can reduce the receiver's dynamic range [10], [11] or just to increase the sensitivity in the range swath of interest [12].

The VCO nonlinearity problem is solved using a correction algorithm designed for wideband nonlinearities that can be described by a polynomial expression. The presence of polynomial nonlinearities leads to a multi-component polynomialphase beat signal. The coefficients of the polynomial-phase signal (PPS) are estimated using the high-order ambiguity function (HAF) [14] on a reference response which can be either a delay line or a high reflectivity target whose propagation delay should be roughly known. Afterwards, with the estimated coefficients, the nonlinearity correction function is built and applied through a time resampling procedure [15].

The nonlinearity correction algorithm we propose in this paper differs from previous works in two ways. On one hand, a typical nonlinearity estimation method (used for example in Vossiek's work [7]) based on the determination of the instantaneous phase of a precision radar reference path is valid only for a single component response, while the HAFbased estimation can extract the nonlinearity coefficients from a multi-component response if there is one highly reflective target relative to other scatterers. On the other hand, the correction method proposed in [4] by Meta et al. requires a multiplication between the beat frequency signal and the nonlinearity term from the transmitted waveform. This implies an oversampling of the beat signal in order to satisfy the Nyquist condition for the nonlinearity term bandwidth and consequently this method is not well suited for large band- 
width nonlinearities (up to gigahertz). In our work, both the estimation and the correction of the nonlinearity are made using only the beat frequency signal, so the bandwidth of the nonlinear term from the transmitted signal doesn't impose the sampling rate.

The X-band FMCW radar is tested with a number of range profiles and SAR images of a few short-range metallic targets. The displacements are computed from the interferometric phase and their absolute errors are below $0.1 \mathrm{~mm}$ in all tests.

This paper is organized as follows. In Section II the shortrange FMCW radar architecture is described. Section III presents the wideband nonlinearity correction algorithm. The polynomial-phase signal model is introduced first. Then, the HAF-based estimation method of the FMCW signal coefficients is discussed in Section III-A and the time resampling correction procedure is exposed in Section III-B. Simulation results prove the efficiency of the proposed correction algorithm in Section III-C. Section IV presents the measurement results: Section IV-A shows the validation of the nonlinearity correction algorithm and in Section IV-B the displacement measurements are presented. Finally, the conclusions are stated in Section V.

\section{SHORT-RANGE FMCW RADAR ARCHITECTURE}

The block diagram of the X-band short-range FMCW radar is shown in Fig. 1. The sweep signal is a linear tuning voltage with $100 \mathrm{~ms}$ period obtained from the signal generator of an USB oscilloscope. The discrete nature of the command signal leads to a stepped frequency modulation with an unambiguous beat frequency inversely proportional to the step duration [16], [17]. The resolution of the signal generator is 12 bits which for the maximum bandwidth of $4 \mathrm{GHz}$ leads to an unambiguous range of over $100 \mathrm{~m}$ (where the behavior is FMCW type). The RF VCO block is a low-cost X-band VCO with $15 \%$ linearity (defined as the ratio between the maximum frequency deviation of the characteristic from linear and the total sweep bandwidth [18], [19]) which provides the local oscillator (LO) signal. The high nonlinearity of this VCO can be software corrected and is no need of an expensive YIG-based VCO. For a linear tuning voltage, the RF VCO signal in a sweep period $T_{p}$ can be written as:

$$
s_{L O}(t)=\cos \left[2 \pi\left(f_{0} t+\frac{1}{2} \alpha_{0} t^{2}\right)+\Phi_{n l}(t)\right],
$$

where $\Phi_{n l}(t)$ is the nonlinearity phase term. The intermediary frequency (IF) block is a direct digital synthesizer with adjustable frequency $(50-250 \mathrm{kHz})$. The transmitted signal consists of two different signals obtained by mixing the LO signal with the IF signal:

$$
\begin{aligned}
& s_{T}(t)=\frac{1}{2} \cos \left[2 \pi\left(\left(f_{0}+f_{I F}\right) t+\frac{1}{2} \alpha_{0} t^{2}\right)+\Phi_{n l}(t)\right]+ \\
& \frac{1}{2} \cos \left[2 \pi\left(\left(f_{0}-f_{I F}\right) t+\frac{1}{2} \alpha_{0} t^{2}\right)+\Phi_{n l}(t)\right] .
\end{aligned}
$$

A part of the transmitted signal gets directly to the mixer from the receiver section through the couplers $(\mathrm{C} 1$ and $\mathrm{C} 2)$ and the delay line. This reference path is used as power level reference and for calibrating the radar with the nonlinearity correction algorithm. In the receiver section, the reflected signal that comes from $N$ different targets is a sum of delayed and attenuated versions of the transmitted signal $s_{T}(t)$ :

$$
s_{R}(t)=\sum_{i=1}^{N} A_{i} s_{T}\left(t-\tau_{i}\right)
$$

where $\tau_{i}$ and $A_{i}$ are the propagation delay and amplitude corresponding to target $i$. The received signal is mixed with the LO and the resulting low frequency signal gets centered around the IF:

$$
\begin{aligned}
& s_{I F}(t)=\frac{1}{4} \sum_{i=1}^{N} A_{i}\left\{\operatorname { c o s } \left[2 \pi \left(\left(f_{I F}+\alpha_{0} \tau_{i}\right) t+\right.\right.\right. \\
& \left.\left.\left(f_{0}-f_{I F}\right) \tau_{i}-\frac{1}{2} \alpha_{0} \tau_{i}^{2}\right)+\left(\Phi_{n l}(t)-\Phi_{n l}\left(t-\tau_{i}\right)\right)\right]+ \\
& \cos \left[2 \pi\left(\left(f_{I F}-\alpha_{0} \tau_{i}\right) t-\left(f_{0}+f_{I F}\right) \tau_{i}+\frac{1}{2} \alpha_{0} \tau_{i}^{2}\right)-\right. \\
& \left.\left.\left(\Phi_{n l}(t)-\Phi_{n l}\left(t-\tau_{i}\right)\right)\right]\right\} .
\end{aligned}
$$

For short-range applications the delay is very small compared to the sweep period. In consequence the residual video phase (RVP) term [20] can be neglected and the nonlinearity phase term difference can be approximated with the derivative multiplied with the delay. Under these assumptions, the IF signal can be rewritten as:

$$
\begin{aligned}
& s_{I F}(t) \approx \frac{1}{4} \sum_{i=1}^{N} A_{i}\left\{\operatorname { c o s } \left[2 \pi \left(\left(f_{I F}+\alpha_{0} \tau_{i}\right) t+\right.\right.\right. \\
& \left.\left.\left.\left(f_{0}-f_{I F}\right) \tau_{i}\right)+\tau_{i} \Phi_{n l}^{\prime}(t)\right)\right]+\cos \left[2 \pi \left(\left(f_{I F}-\alpha_{0} \tau_{i}\right) t-\right.\right. \\
& \left.\left.\left.\left.\left(f_{0}+f_{I F}\right) \tau_{i}\right)-\tau_{i} \Phi_{n l}^{\prime}(t)\right)\right]\right\} .
\end{aligned}
$$

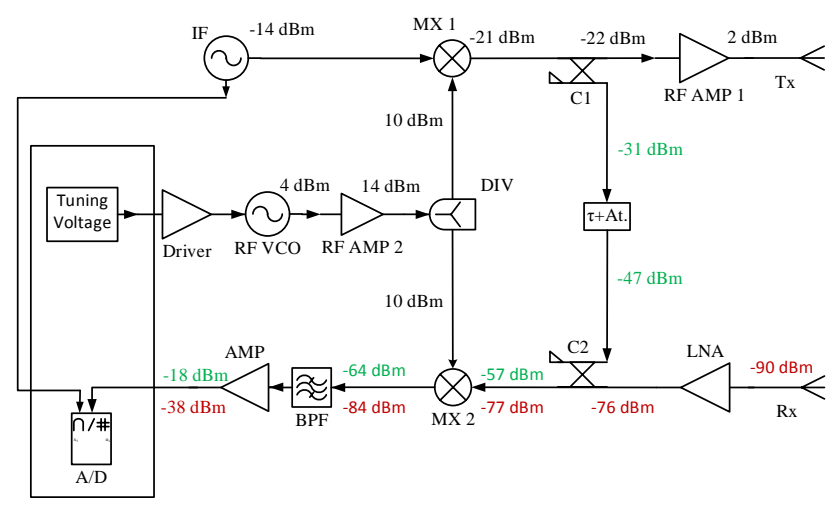

Fig. 1. Block diagram of the X-band FMCW radar.

This signal is filtered, amplified and afterwards sampled with $1 \mathrm{MHz}$ sampling rate. The IF signal spectrum consists of two groups of spectral components placed symmetrically around the intermediary frequency as presented in Fig. 2. The analog band-pass filter (BPF: 25-500 kHz bandwidth) removes 
TABLE I

SPECIFICATIONS OF THE FMCW RADAR SYSTEM

\begin{tabular}{|c|c|}
\hline Maximum bandwidth & $8-12 \mathrm{GHz}$ \\
\hline Sweep period & $100 \mathrm{~ms}$ \\
\hline IF frequency & $50-250 \mathrm{kHz}$ \\
\hline VCO linearity & $15 \%$ \\
\hline Tuning signal & Triangular/Sawtooth \\
\hline Transmitted power & $2 \mathrm{dBm}$ \\
\hline Antenna gain & $17 \mathrm{dBi}$ \\
\hline Beamwidth (azimuth/elevation) & $25^{\circ} / 25^{\circ}$ \\
\hline Maximum range & $100 \mathrm{~m}$ \\
\hline
\end{tabular}

the low-frequency components (resulted from self-mixing and local oscillator leakage in the transmitted signal), improves the signal to noise ratio by reducing the noise bandwidth and acts as anti-aliasing filter. Additional digital filters can be applied to select an imposed range interval before mixing to baseband. The specifications of the wideband FMCW radar system are summarized in Table I.

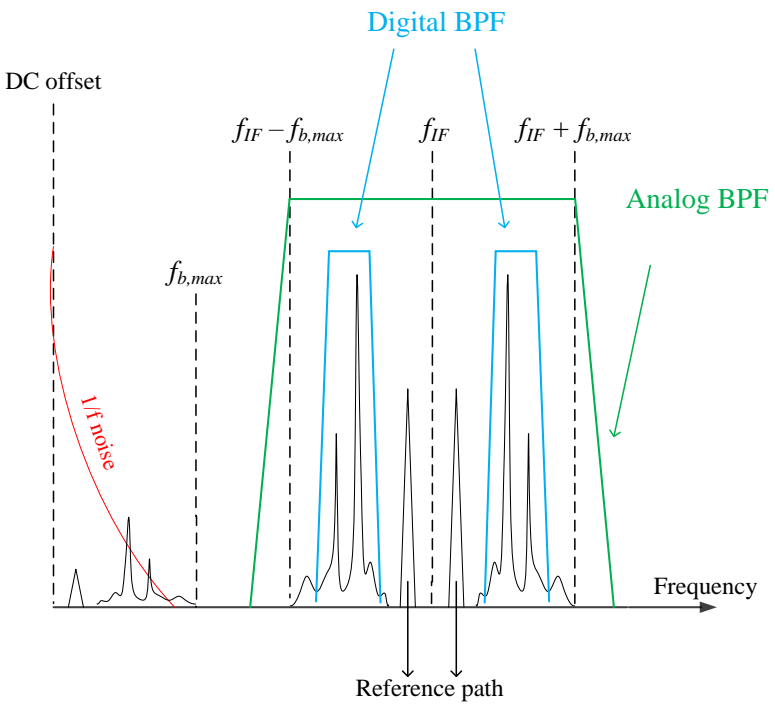

Fig. 2. Intermediary frequency signal spectrum. The analog filter removes the low-frequency components, improves the signal to noise ratio and acts as anti-aliasing filter.

In order to shift the spectrum in baseband, the signal in (5) should be digitally multiplied with the sampled IF sinusoidal signal. The resulting beat frequency signal is (neglecting the 0.5 factors resulted from cosine multiplication):

$$
s_{b}(t)=\sum_{i=1}^{N} A_{i} \cos \left[2 \pi\left(f_{0}+\alpha_{0} t+\Phi_{n l}^{\prime}(t)\right) \tau_{i}\right] .
$$

If the range profile is computed as the Fourier transform of this beat signal, the nonlinearity terms spread the energy of each target and the resolution gets deteriorated. In order to avoid this shortcoming, the beat signal is processed with the nonlinearity correction algorithm described in the following section. Notice that the same beat signal as the one in (6) would be obtained for a homodyne architecture if we neglect the undesired low-frequency components, so the proposed nonlinearity correction algorithm can be applied to both heterodyne and homodyne architectures.

Note that in contrast to other implementations (like [13]) the proposed architecture has certain differences which start with the main reason for using the intermediary frequency (to avoid the homodyne problems, not to build a range-gate). Using a relatively large sweep period leads to a smaller frequency interval of the expected beat frequencies which implies a reduced noise bandwidth after the weighting window applied in computing the range profile (the equivalent noise bandwidth is around $15 \mathrm{~Hz}$ for $100 \mathrm{~ms}$ sweep period). Another observation is that both groups of spectral components around the IF are mixed to baseband and cumulated (not only one lateral sideband) which avoids using an image reject filter.

\section{NONLINEARITY CORRECTION ALGORITHM}

The slope of the frequency-voltage characteristic for some radio frequency VCOs may be reasonably approximated by a quadratic curve [19]. However, a more general approach is to assume a polynomial frequency-voltage dependence. This means that the nonlinearity phase term can be expressed as:

$$
\Phi_{n l}(t)=2 \pi \sum_{k=2}^{K} \frac{\beta_{k}}{k+1} t^{k+1} .
$$

With this assumption, by introducing (7) in (6) the beat signal becomes a PPS:

$$
s_{b}(t)=\sum_{i=1}^{N} A_{i} \exp \left[j 2 \pi\left(f_{0}+\alpha_{0} t+\sum_{k=2}^{K} \beta_{k} t^{k}\right) \tau_{i}\right] .
$$

The correction algorithm proposed in this paper aims to turn this multi-component PPS into a sum of $N$ complex sinusoids. In this way each target appears as a sinc function in the range profile. The algorithm consists of two steps: the estimation of the FMCW signal coefficients (linear chirp rate $\alpha_{0}$ and nonlinearity coefficients $\beta_{k}$ ) using the high-order ambiguity function and the correction of the beat signal by time resampling.

\section{A. Estimation of the FMCW signal coefficients}

The estimation is based on the presence of a reference target response (with amplitude $A_{r e f}$ and propagation delay $\tau_{r e f}$ ) in the FMCW signal. This particular PPS component can be extracted by bandpass filtering the beat signal around the beat frequency corresponding to $\tau_{r e f}$. The filtered signal can be written as:

$$
s_{b, f}(t)=s_{b, f}\left(t, \tau_{r e f}\right)+\sum_{m=1}^{M} s_{b, f}\left(t, \tau_{m}\right) .
$$

where $M$ is the number of significant PPS components located near the reference response in the filter's pass band which cannot be eliminated. In the estimation, it is considered that the reference target is highly reflective relative to the remaining $M$ 
components. Although the filtered signal has other components besides the reference response, the FMCW signal coefficients can be estimated using the high-order ambiguity function due to its ability to deal with multiple component PPS's if the highest order phase coefficients of the components are not the same [21], [22]. This happens for the FMCW signal because each component is linked to a target with a certain propagation delay.

The starting point is the high-order instantaneous momentum (HIM), which can be defined for a signal $s(t)$ as [14]:

$$
H I M_{k}[s(t) ; \tau]=\prod_{i=0}^{k-1}\left[s^{(* i)}(t-i \tau)\right]^{\left(\begin{array}{c}
k-1 \\
i
\end{array}\right)},
$$

where $k$ is the HIM order, $\tau$ is the lag and $s^{(* i)}$ is an operator defined as:

$$
s^{(* i)}(t)= \begin{cases}s(t) & \text { if } i \text { is even, } \\ s^{*}(t) & \text { if } i \text { is odd, }\end{cases}
$$

where $i$ is the number of conjugate operator "*" applications. The high-order ambiguity function (HAF) is defined as the Fourier transform of the HIM.

If we assume a PPS model for the analyzed signal, i.e.:

$$
s_{P P S}(t)=A \exp \left[j 2 \pi \sum_{m=0}^{k} a_{m} t^{m}\right],
$$

the essential property of the HIM is that, the $k$-th order HIM is reduced to a harmonic with amplitude $A^{2^{k-1}}$, frequency $\tilde{f}_{k}$ and phase $\Phi_{k}$ :

$$
H I M_{k}\left[s_{P P S}(t) ; \tau\right]=A^{2^{k-1}} \exp \left[j\left(2 \pi \tilde{f}_{k} t+\Phi_{k}\right)\right],
$$

where

$$
\tilde{f}_{k}=k ! \tau^{k-1} a_{k} .
$$

So the HAF of this HIM should have a spectral peak at the frequency $\tilde{f}_{k}$. Based on this result, an algorithm that estimates sequentially the coefficients $a_{k}$ was proposed in [23]. Starting with the highest order coefficient, at each step, the spectral peak is determined, and an estimation value $\hat{a_{k}}$ of $a_{k}$ is computed from (14). With this value, the phase term of the higher order is removed:

$$
s_{P P S}{ }^{(k-1)}(t)=s_{P P S}{ }^{(k)}(t) \exp \left(-j 2 \pi \hat{a_{k}} t^{k}\right)
$$

and the procedure repeats iteratively. A classical problem of this nonlinear method is the propagation of the approximation error from one higher order to the lower ones, but in the case of typical frequency-voltage VCO characteristics this effect is not critical because an approximation order of only 3 or 4 is required. Still, if a higher order is necessary a warpedbased polynomial order reduction as described in [24] could be employed.

After applying this iterative algorithm to the FMCW reference signal and obtaining the polynomial phase coefficients, the linear chirp rate and the nonlinearity coefficients can be computed as:

$$
\begin{aligned}
\alpha_{0} & =\frac{\hat{a_{1}}}{\tau_{r e f}} \\
\beta_{k} & =\frac{\hat{a_{k}}}{\tau_{r e f}}, k=\overline{2, K} .
\end{aligned}
$$

\section{B. Time Resampling}

Due to the fact that the frequency-voltage characteristic of a VCO is monotonous, for a linear voltage sweep the resulting polynomial phases of the beat signal components are monotonous functions for $t \in\left[0, T_{p}\right]$. Therefore, the beat signal in (8) can be rewritten as:

$$
s_{b}(t)=\sum_{i=1}^{N} A_{i} \exp \left\{j 2 \pi\left[f_{0}+\alpha_{0} \theta(t)\right] \tau_{i}\right\}, t \in\left[0, T_{p}\right],
$$

where

$$
\theta(t)=t\left(1+\sum_{k=2}^{K} \frac{\beta_{k}}{\alpha_{0}} t^{k-1}\right)
$$

is a monotonous function of time $t$, which can be interpreted as a new time axis. Hence, if the time axis is changed to $\theta$, the beat signal becomes a sum of $N$ complex sinusoids, which was the purpose of the correction algorithm. Moreover, in the context of radar detection, the highly correlated clutter from a nonlinear range profile gets decorrelated in a range profile computed for the new time axis.

Notice that in the definition of $\theta$ the nonlinearity coefficients $\beta_{k}$ are normalized to the linear chirp rate $\alpha_{0}$ which means that the exact value of the reference propagation delay is not needed. However, a rough value is required for the estimation part in order to extract the reference response.

From the implementation point of view, the beat signal is a digital signal $s_{b}[n]$ uniformly sampled at the moments $t_{n}$, $n=\overline{0, N_{s}-1}$, where $N_{s}$ is the number of samples. However, the samples $s_{b}[n]$ of the beat signal related to the moments $\theta_{n}=\theta\left(t_{n}\right)$ of the $\theta$ time axis lead to a non-uniformly sampled signal. It can be shown that the average sampling interval of $\theta$ is:

$$
\overline{\theta_{S}}=\frac{\bar{\alpha}}{\alpha_{0}} T_{s},
$$

where $\bar{\alpha}$ is the mean chirp rate and $T_{s}$ the uniform sampling interval. According to [25], for a nonuniformly sampled signal, the average sampling rate must respect the Nyquist condition. For $\bar{\alpha}>\alpha_{0}$, this condition can be fulfilled if the beat signal is oversampled (the chirp rate in the origin and the average chirp rate typically have the same order of magnitude, so an oversampling of at most 10 is enough). If the signal is aliasfree, it can be resampled with an interpolation procedure (e.g. with spline functions) in order to obtain a uniformly sampled signal in relation with the $\theta$ time axis. Afterwards, the range profile is computed by applying the discrete Fourier transform to the resampled signal. The nonlinearity correction algorithm is summarized in the block diagram from Fig. 3. 


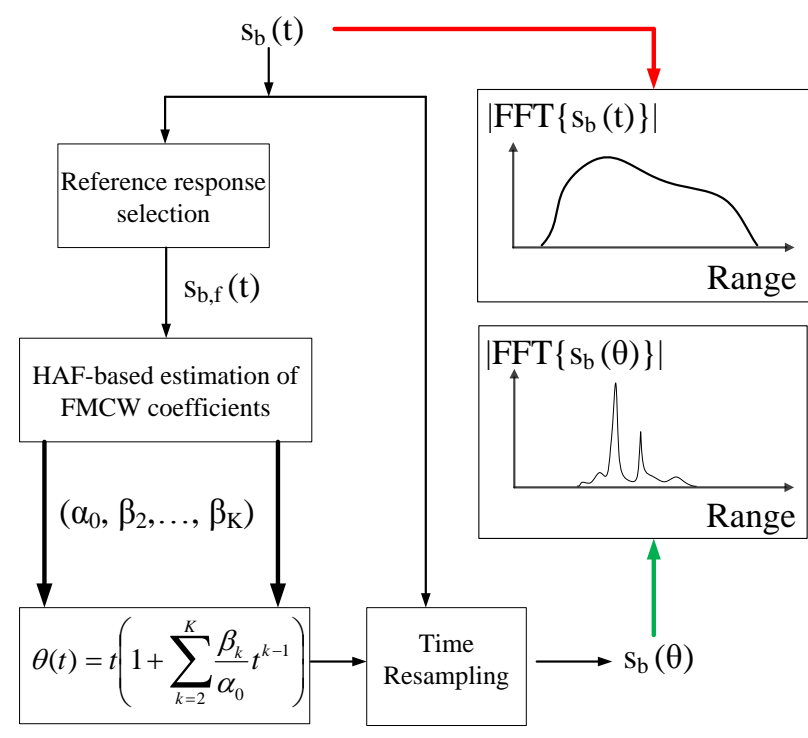

Fig. 3. Wideband nonlinearity correction algorithm based on high-order ambiguity functions and time resampling.

\section{Nonlinearity Correction Algorithm Simulation}

The range profiles of a FMCW radar based on an X-band VCO with $15 \%$ linearity were simulated. The chirp bandwidth was $4 \mathrm{GHz}$, the sweep rate $50 \mathrm{~Hz}$ and the sampling frequency $1 \mathrm{MHz}$. The responses of six stationary targets with different amplitudes were considered. The reference target was the one located at $50 \mathrm{~m}$. The nonlinear and corrected range profiles are shown in Fig. 4. In the nonlinear range profile the targets can't be distinguished due to the overlapping of the frequency spread responses of each target. The correction algorithm enhances the $-3 \mathrm{~dB}$ resolution up to the theoretical limit (around 4.9 $\mathrm{cm}$ for a Hamming window), although in the filtered signal used for estimating the FMCW coefficients there are four other targets close to the reference response. This result is in keeping with the capability of the HAF estimation method to extract the maximum amplitude PPS component if the ratio to the other components is above a certain threshold (around $10 \mathrm{~dB}$ ). Besides the dramatically enhanced resolution, the correction algorithm improves the peak level of each target which leads to an increase of the signal to noise ratio with over $20 \mathrm{~dB}$.

\section{MEASUREMENT RESULTS}

This section is divided in two parts: the first part validates the wideband nonlinearity correction algorithm on real data and the second one is concerned with displacement measurements using the interferometric phase derived from range profiles and SAR images.

\section{A. Nonlinearity Correction Algorithm Validation}

The nonlinearity correction algorithm was first tested using only the transceiver of the implemented radar system and having artificial targets obtained with delay lines and attenuators. The tuning voltage versus frequency calibration curve

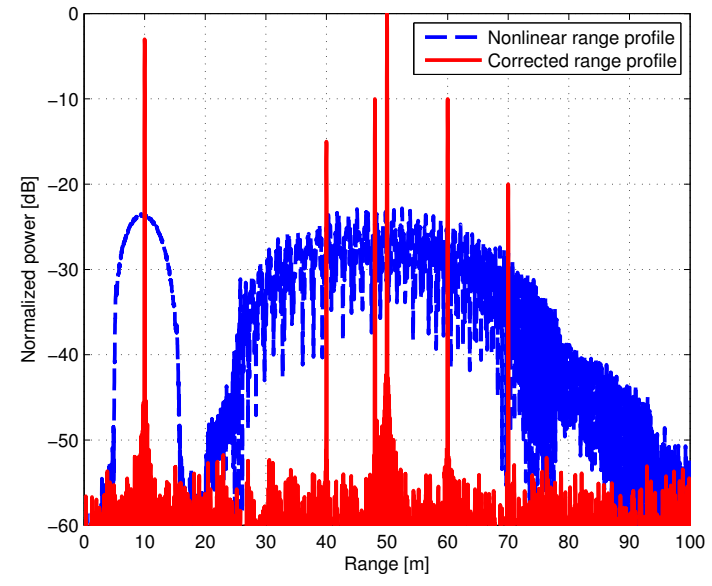

(a)

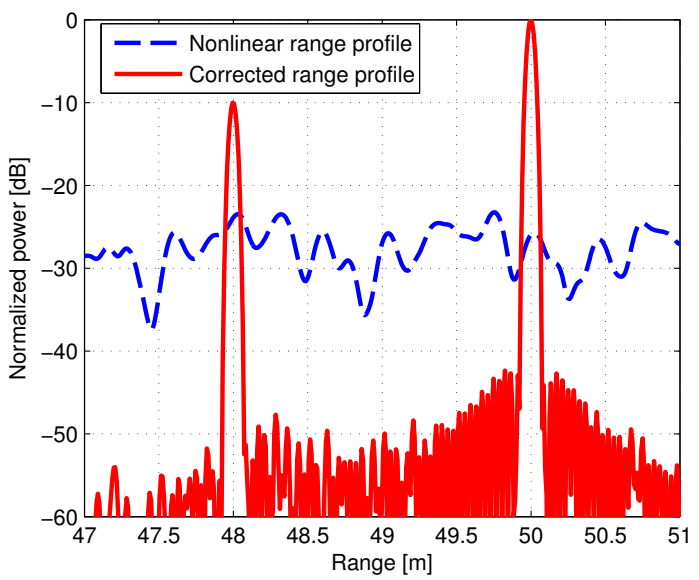

(b)

Fig. 4. Range profiles simulation results: (a) 0-100m range, (b) detailed $47-51 \mathrm{~m}$ range. In the nonlinear profile five targets are completely indistinguishable. The corrected profile leads to a resolution near the theoretical limit and improves the signal to noise ratio.

of the VCO was measured. The range profiles obtained with a predistorted command signal based on the calibration curve were considered as reference. A few data sets were collected under the same external conditions as for the calibration curve measurement. Two delay lines having air-equivalent lengths of $30 \mathrm{~cm}$ (short path) and respectively $240 \mathrm{~cm}$ (long path) were used as targets. The chirp bandwidth was $4 \mathrm{GHz}$ and the sweep interval $100 \mathrm{~ms}$. The correction was done using both lines in order to analyze the influence of the reference response delay on the algorithm's performance. For a 4th order polynomial approximation, the HAFs plots of the FMCW coefficients in both cases are shown in Fig. 5. The estimated coefficients were used to generate nonlinearity corrected beat signals by employing the time resampling procedure. The range profiles were obtained by applying a Hamming window before the Fourier transform.

Fig. 6 shows a comparison between the range profiles obtained for the two delay lines in different cases and the range profile obtained for the predistorted sweep (in each comparison the range profiles are normalized such that the short path peak responses have the same level). The range 

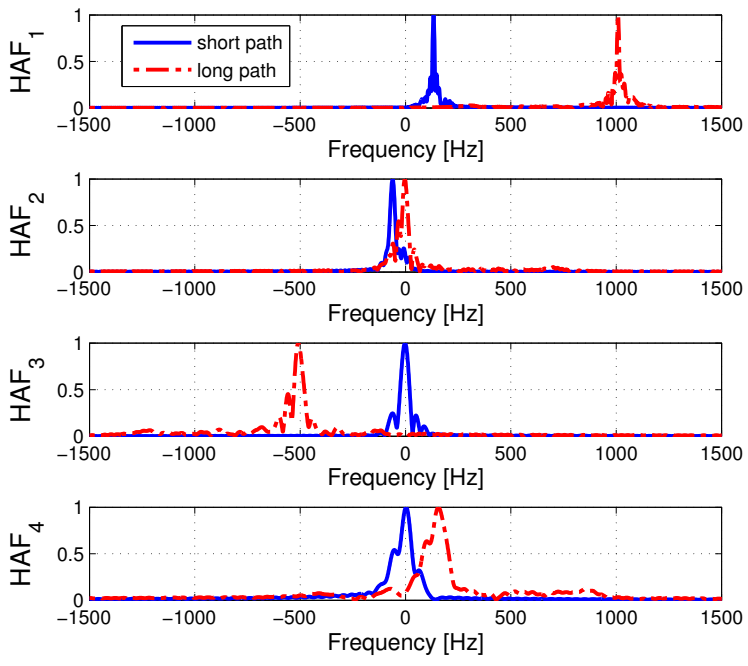

Fig. 5. High-order ambiguity functions of the FMCW signal for two delay lines. The continuous plots show the functions for a $30 \mathrm{~cm}$ air-equivalent length delay line (short path) and the dotted ones for a $240 \mathrm{~cm}$ air-equivalent delay line (long path).

profile for a linear sweep is presented in Fig. 6a. The energy of both targets is highly spread in frequency and the main lobe for the long path occupies more than 40 resolution cells (expected in view of the high degree of nonlinearity of the VCO). The range profile corrected using the short path coefficients (shown in Fig. 6b) is similar to the predistorted sweep range profile for the short path response, but the energy of the long path is still spread and there are two main lobes for the same target. This effect is linked with the HAFs for the high-order nonlinearity terms ( 3 and 4$)$ in the short reference path case which are hardly noticeable and can't be estimated properly. However, the long path calibration range profile from Fig. $6 \mathrm{c}$ is very similar to the one obtained with the predistorted sweep, so this software nonlinearity correction method provides good results if the calibration path is long enough to emphasize the nonlinearities (the higher order terms to be highlighted and properly estimated). A clear advantage of the proposed correction algorithm compared to the predistorted sweep technique is that the FMCW signal coefficients used for correction can be computed for each sweep and can include various frequency drifts (due to temperature, frequency pushing, etc.). The results of the range profiles comparison are summarized in Table II where the $-10 \mathrm{~dB}$ resolutions are computed for both targets. Although the resolution for the long path corrected range profile is better than for the predistorted sweep range profile there are still present some residual nonlinearities (deterministic as well as random) which have small bandwidths and whose effect increases with range. However, they can be further mitigated with methods like those presented in [4], [7], [26].

In order to validate the HAF-based nonlinearity estimation method for a multi-component response, a data set was acquired for a scene containing three main scatterers: one highly reflective metal disc and two vertical metal bars. The range profiles obtained in this case are shown in Fig. 7. The nonlinearity coefficients are computed on the 1.2-5.2 m range
TABLE II

NONLINEARITY CORRECTION ALGORITHM - 10 DB RESOLUTION

\begin{tabular}{|c||c|c|}
\hline & $\begin{array}{c}\text { Short Path } \\
\text { Resolution }(\mathrm{cm})\end{array}$ & $\begin{array}{c}\text { Long Path } \\
\text { Resolution }(\mathrm{cm})\end{array}$ \\
\hline Predistorted sweep & 8.18 & 8.47 \\
\hline Short path correction & 8.23 & 19.87 \\
\hline Long path correction & 8.17 & 8.45 \\
\hline
\end{tabular}

interval taking as reference target the metal disc. While on the initial nonlinear range profile obtained for the linear voltage sweep appears only a large continuous target, on the corrected profile the three targets are clearly highlighted. Notice that the power reflected by the metal disc is more than $10 \mathrm{~dB}$ higher in comparison to the other scatterers which is in agreement with the HAF method applicability threshold.

In the following part we show the results of the software nonlinearity correction algorithm applied to FMCW radar data acquired for a frequency sweep from 8 to $11 \mathrm{GHz}$. In Fig. 8 are shown the range profiles obtained before and after applying the correction algorithm for a scene containing a corner reflector (situated at $2.5 \mathrm{~m}$ from the radar) which was used as reference target for the nonlinearity estimation. In the corrected range profile the main lobe of the corner reflector target reaches the theoretical $-3 \mathrm{~dB}$ resolution for a $3 \mathrm{GHz}$ bandwidth and Hamming window (around $6.5 \mathrm{~cm}$ ).

Fig. 9 presents the results obtained from applying the nonlinearity correction for a synthetic aperture image acquired by moving the FMCW radar on a $30 \mathrm{~cm}$ rail. The scene in the synthesized image contained some metal bars and one highlyreflective metal disc. The software nonlinearity correction was employed by resampling each line of the initial image before applying the matched filter algorithm [27] to obtain the synthetic aperture radar (SAR) image. Notice that in the image from Fig. 9a the targets can't be distinguished while in the corrected version they are clearly range focused (Fig. 9b).

\section{B. Displacement Measurements}

A set of simulations based on the FMCW platform's parameters was carried out in order to determine the expected theoretical accuracy of the displacement measurements. In each simulation were generated two noisy and nonlinearity corrected range profiles containing one and the same target with a given radar cross section. The target's distance from the radar in the two range profiles differed with $1 \mathrm{~mm}$. The measured displacement was the one obtained from the phase difference of the target's complex amplitudes in the two range profiles. The error was computed as the difference between the $1 \mathrm{~mm}$ real value and the measured one. For each simulation the signal to noise ratio (SNR) was computed as the ratio between the target's peak response and the noise floor value (which for a range profile is the noise power computed in the equivalent noise bandwidth of the weighing window used before the Fourier transform). For each SNR value, the error's dispersion was computed over 1000 realizations of the two range profiles. The relationship between the displacement error 


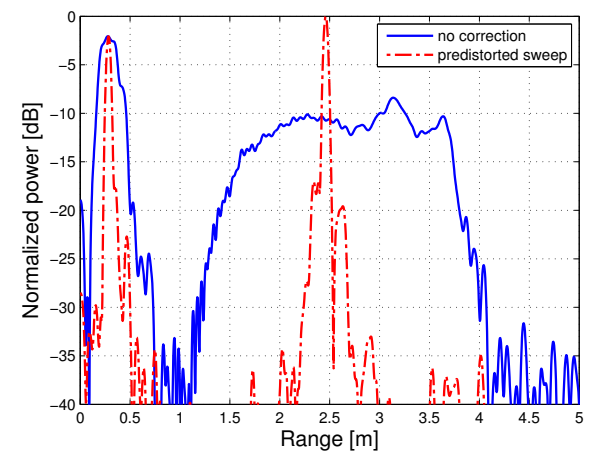

(a)

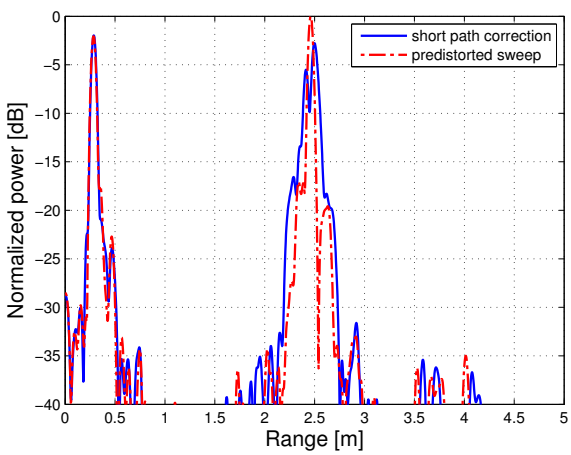

(b)

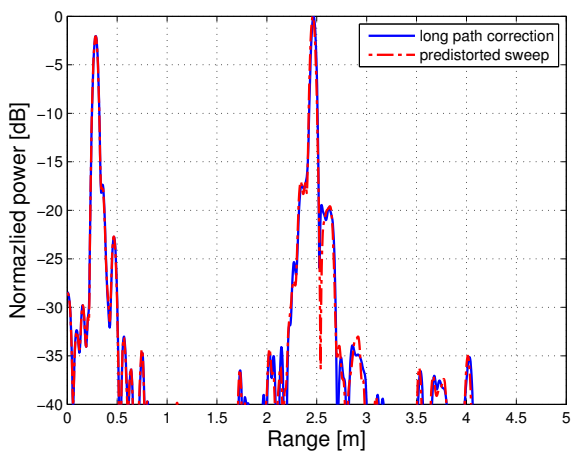

(c)

Fig. 6. Range profiles for two delay lines. The profiles obtained with a linear sweep and software nonlinearity correction are compared with the profile resulted for a predistorted sweep (shown with dotted line) computed from the measured frequency-voltage calibration curve. There are three cases considered: (a) no correction, (b) short path correction, (c) long path correction.

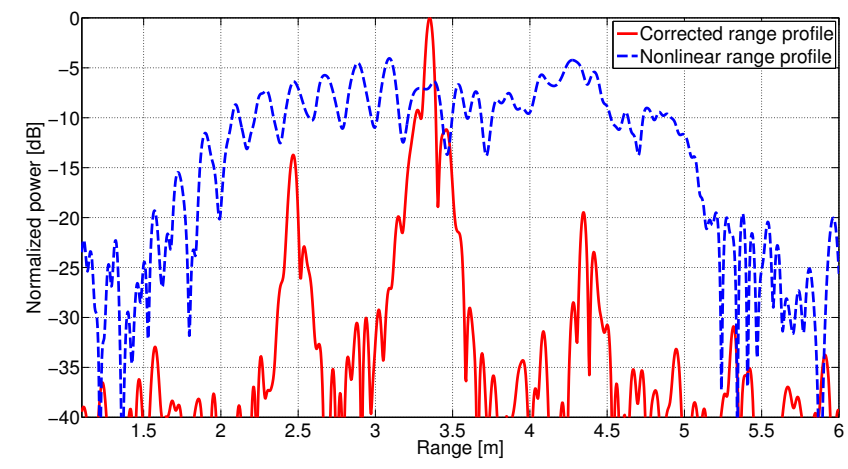

Fig. 7. Experimental range profiles for a scene containing two metal bars and one highly reflective metal disc. On the corrected range profile the three targets are clearly separated, while in the nonlinear range profile the scatterers can't be distinguished.

dispersion and the SNR for targets placed at $2 \mathrm{~m}$ and $50 \mathrm{~m}$ is plotted in Fig. 10. Notice that for SNRs greater than $25 \mathrm{~dB}$ the displacement measurement error should stay below $0.1 \mathrm{~mm}$.

The displacement measurements with the FMCW radar were made using both range profiles and synthetic aperture images for the $3 \mathrm{GHz}$ bandwidth. The results are presented in the following sections.

1) Range profiles based displacements: Different targets such as metal bars and corner reflectors were placed in front of the radar at various ranges (1-6 m). The displacement measurements were conducted as follows. One target was placed at a certain distance from the radar and the range profile was computed after cumulating the received signal over 10 sweep periods. Afterwards, the target was displaced with a few millimeters using a caliper based device (with $0.02 \mathrm{~mm}$ accuracy) bonded to the target and another range profile was obtained. Due to the very small displacement in range, the target will most likely be in the same range bin in both measurements, but the phase from that bin differs proportionally with the displacement. If the range bin is not the same and the displacement is unambiguous (e.g. is lower than half the wavelength corresponding to the central frequency), the displacement is still obtained as a phase difference, but between the phases of the corresponding range bins from

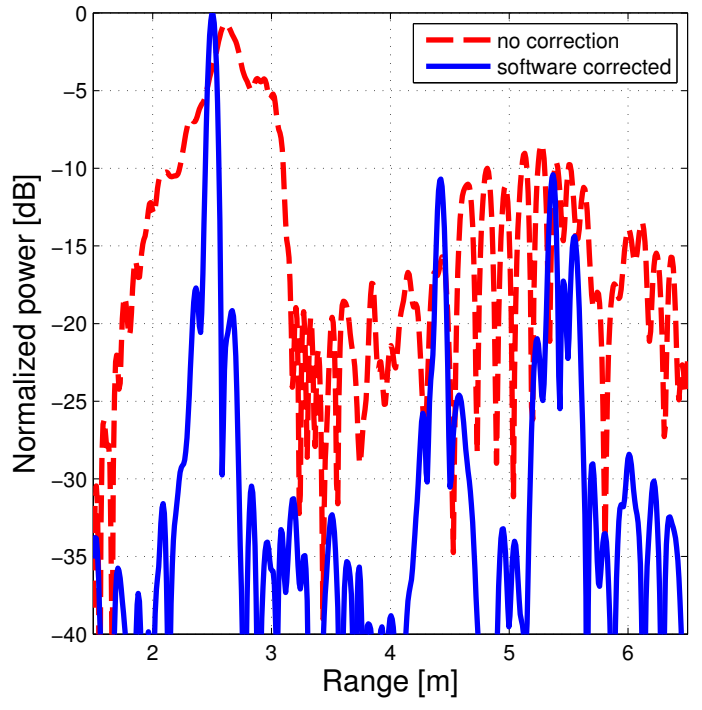

Fig. 8. Range profile for an indoor scene containing a corner reflector (at 2.5 $\mathrm{m}$ ) used as reference target for the software nonlinearity correction method. The chirp bandwidth was $3 \mathrm{GHz}$ and the sweep interval $100 \mathrm{~ms}$.

the two acquired range profiles. Anyway, the displacement is evaluated using the interferometric phase $\phi$ of the FMCW complex range profile:

$$
\delta r=\frac{c_{0}}{4 \pi f_{c}} \phi
$$

where $\delta r$ is the displacement, $f_{c}$ the central frequency and $c_{0}$ the speed of light in air. The measured displacements and their corresponding absolute errors in a few measurements are summarized in Fig. 11. The displacements errors are below $0.1 \mathrm{~mm}$ which is in keeping with the SNR of over $30 \mathrm{~dB}$ (as resulted from the range profiles).

2) SAR Images based displacements: The measurement procedure for the SAR images based displacements was similar to the one described in the previous subsection, but the experimental setup was the one used to obtain the nonlinearity corrected SAR image from Fig. 9b. In turn, one of the targets was moved on different directions between image acquisitions and the displacement was projected on the local line of sight 


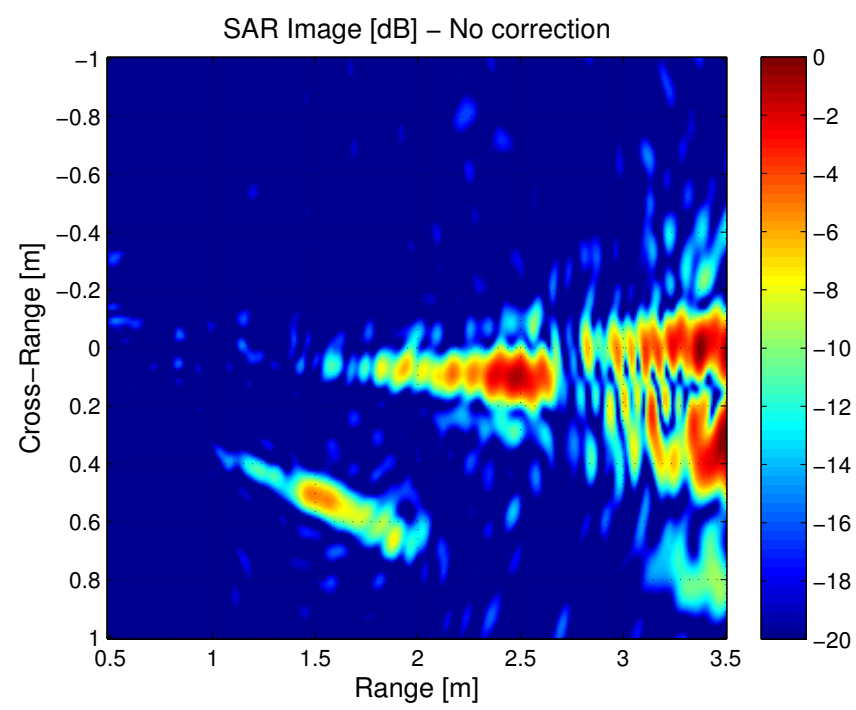

(a)

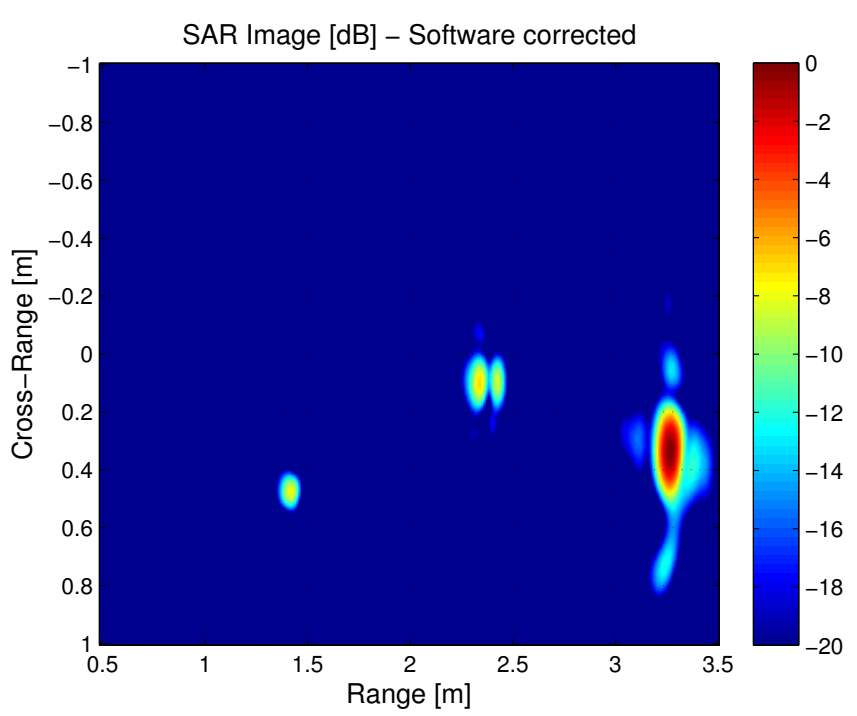

(b)

Fig. 9. Results of the software nonlinearity correction applied to the FMCW image before focusing. In the SAR image affected by the VCO nonlinearity (a) the targets are almost indistinguishable, while in the corrected image (b) the targets are clearly separated.

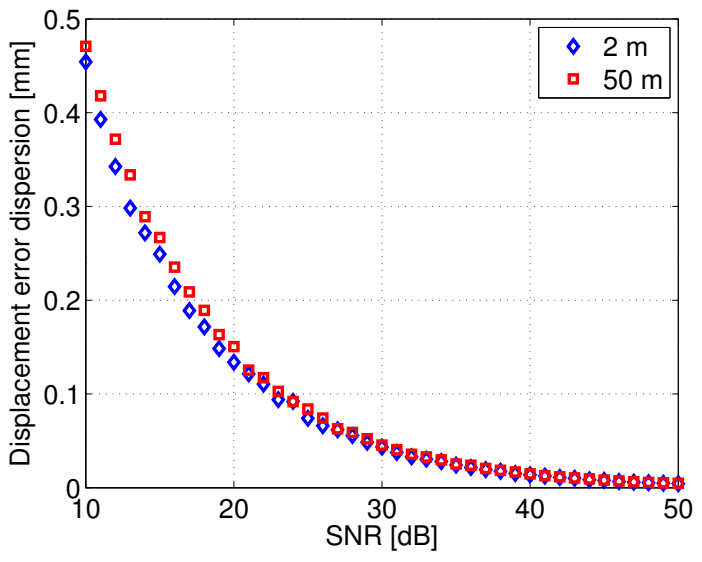

Fig. 10. Simulated displacement error dispersion vs. SNR for targets placed at $2 \mathrm{~m}$ and $50 \mathrm{~m}$. The expected measurement error should get below $0.1 \mathrm{~mm}$ for SNRs higher than $25 \mathrm{~dB}$.

(LOS) direction. Due to the short range the projection angle was different from one target to another.

The complex correlation coefficient $c=\rho \exp (j \phi)$ was computed for each pair of SAR images as [28]:

$$
c=\frac{\sum_{i=1}^{L} z_{1 i} z_{2 i}^{*}}{\sqrt{\sum_{i=1}^{L}\left|z_{1 i}\right|^{2}} \sqrt{\sum_{i=1}^{L}\left|z_{2 i}\right|^{2}}},
$$

where $L$ is the number of samples used for spatial averaging and $z_{1 i}, z_{2 i}$ are the complex numbers (pixels) corresponding to sample $i$. The magnitude $\rho$ of the correlation coefficient is the interferometric coherence and describes the phase stability within the estimation neighborhood [29]. The LOS displacements between two SAR images were computed from the phase of the correlation coefficient (the interferometric phase)
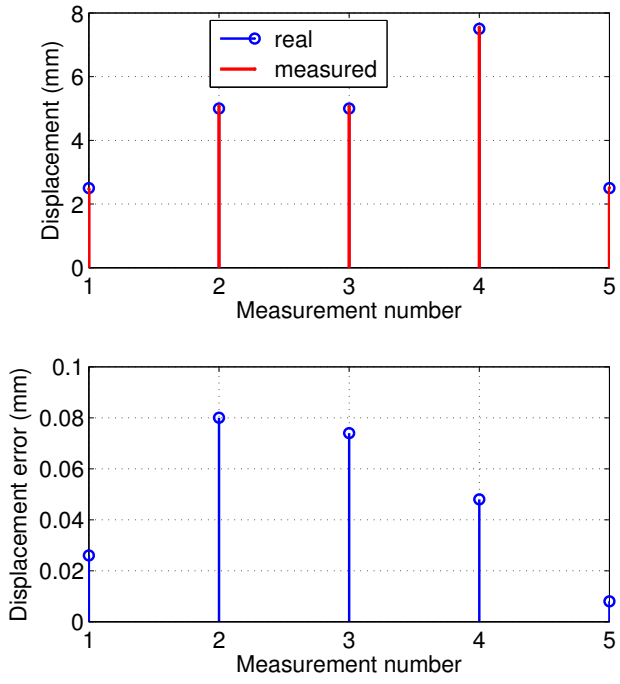

Fig. 11. Range profile displacement measurements results.

using (20). The coherence and interferogram computed for two SAR images with a $3 \times 3$ pixels boxcar are shown in Fig. 12. Notice that in the proximity of the metal targets the coherence is around unity and the phase has approximatively constant value. This is linked to the fact that for the considered experimental setup, there are actually a few scatterers in a resolution cell located in the vicinity of the metal targets which leads to a less perceptible clutter. Fig. 13 shows a few measured LOS displacements and the corresponding absolute errors.

\section{Conclunsions}

In this paper was presented an X-band FMCW radar used for displacements measurement of short-range targets. The radar's transceiver is based on an intermediary frequency 


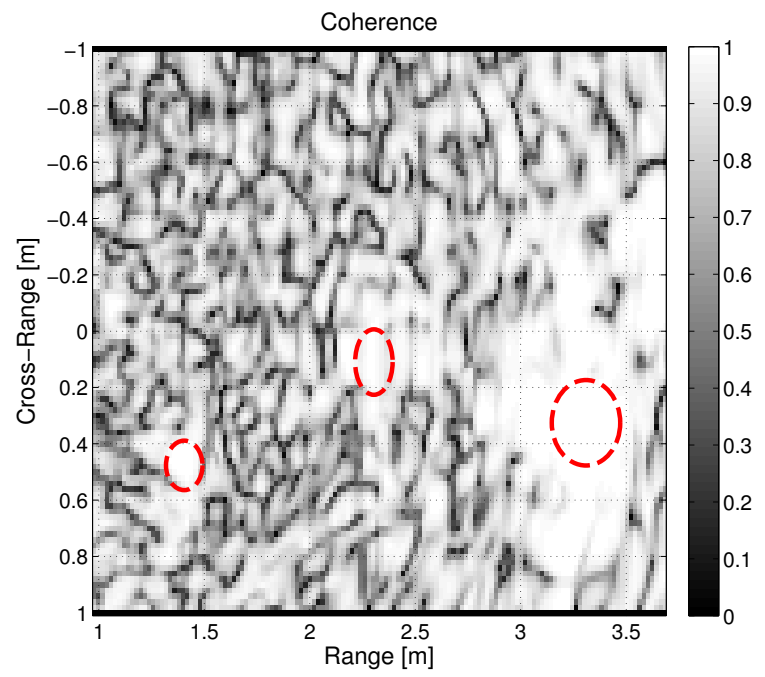

(a)

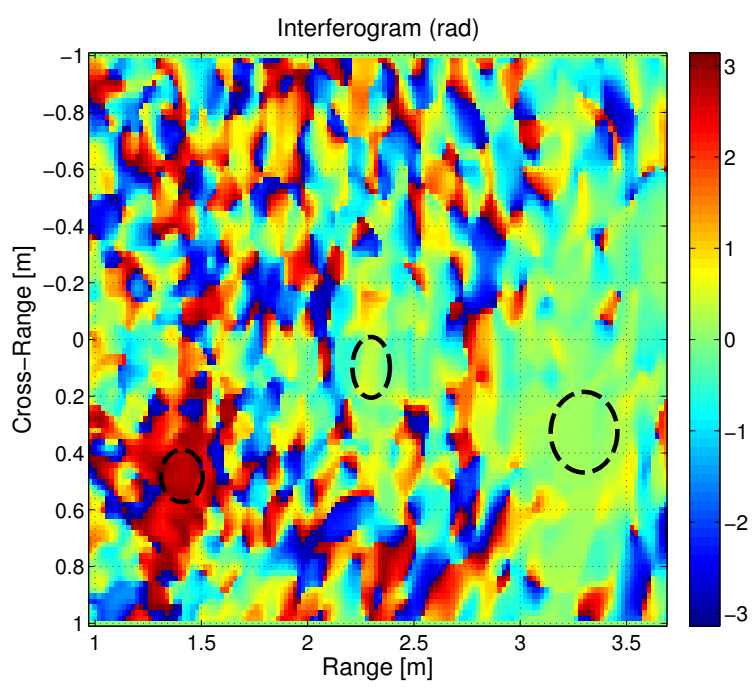

(b)

Fig. 12. Coherence (a) and interferogram (b) of two acquired SAR images computed with a $3 \times 3$ boxcar. The coherence is almost 1 and the phase has approximatively constant value in the proximity of the metal targets (marked by the dotted ellipses).
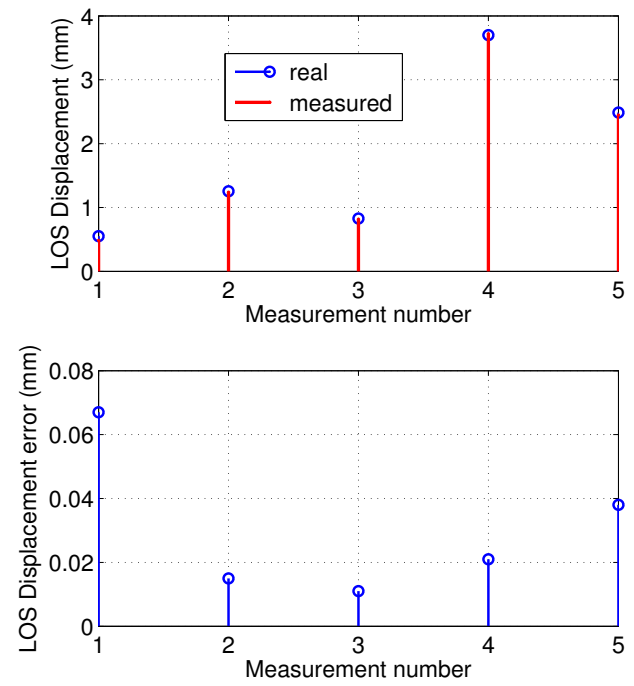

Fig. 13. SAR Image line of sight displacement measurements results.

architecture in order to avoid the specific homodyne problems and enhance the system's sensitivity. The nonlinearity of the VCO was mitigated using a wideband nonlinearity correction algorithm that uses HAF-based estimation and time resampling of the beat signal. The improvements due to the nonlinearity correction are clearly highlighted in the range profiles and the SAR images obtained with the FMCW radar system. The radar's measurement capabilities were validated on the data sets acquired for various targets. The displacements errors were less than $0.1 \mathrm{~mm}$ for metallic targets placed at a few meters from the radar.

\section{ACKNOWLEDGMENT}

The authors would like to thank the Électricité de France (EDF) company for supporting the development of the Xband FMCW radar experimental platform. They would also like to acknowledge the useful suggestions of the anonymous reviewers.

\section{REFERENCES}

[1] A. Meta, P. Hakkart, F. der Zwan, P. Hoogeboom, and L. Ligthart, "First demonstration of an X-band airborne FMCW SAR," in Proc. EuSAR'06, Dresden, Germany, May 2006, pp. 1-4.

[2] G. L. Charvat, "Low-cost, high resolution X-band laboratory radar system for synthetic aperture radar applications," in Proc. IEEE International Conference on Electro/information Technology, East Lansing, MI, USA, May 2006, pp. 529-531.

[3] L. Besser and R. Gilmore, Practical RF Circuit Design for Modern Wireless Systems. London: Artech House, 2003.

[4] A. Meta, P. Hoogeboom, and L. Ligthart, "Range nonlinearities correction in FMCW SAR," in Proc. IGARSS'06, Denver, Denver, USA, Jul. 2006, pp. 403-406.

[5] N. Pohl, T. Jacschke, and M. Vogt, "Ultra high resolution SAR imaging using an $80 \mathrm{ghz}$ FMCW-Radar with $25 \mathrm{ghz}$ bandwidth," in Proc. EuSAR'12, Nuremberg, Germany, Apr. 2012, pp. 189-192.

[6] P. Lowbridge, "A low cost mm-wave cruise control system for automotive applications," Microwave Journal, pp. 24-36, Oct. 1993.

[7] M. Vossiek, P. Heide, M. Nalezinski, and V. Magori, "Novel FMCW radar system concept with adaptive compensation of phase errors," in Proc. EuMC'96, vol. 1, Prague, Czech Republic, Sep. 1996, pp. 135138.

[8] A. Meta, P. Hoogeboom, and L. P. Ligthart, "Signal processing for FMCW SAR," IEEE Trans. Geosci. Remote Sensing, vol. 45, no. 11, pp. 3519-3532, Nov. 2007.

[9] A. Anghel, G. Vasile, R. Cacoveanu, C. Ioana, and S. Ciochina, "Shortrange FMCW X-band Radar Platform For Millimetric Displacements Measurement," in Proc. IGARSS 2013, Melbourne, Australia, Jul. 2013, pp. 1111-1114.

[10] G. L. Charvat, L. C. Kempel, E. J. Rothwell, C. M. Coleman, and E. L. Mokole, "A through-dielectric ultrawideband (uwb) switchedantenna-array radar imaging system," Antennas and Propagation, IEEE Transactions on, vol. 60, no. 11, pp. 5495-5500, 2012.

[11] G. Charvat, L. C. Kempel, E. Rothwell, C. Coleman, and E. Mokole, "A through-dielectric radar imaging system," Antennas and Propagation, IEEE Transactions on, vol. 58, no. 8, pp. 2594-2603, 2010.

[12] G. Charvat, L. Kempell, and C. Coleman, "A low-power high-sensitivity $\mathrm{x}$-band rail sar imaging system [measurement's corner]," Antennas and Propagation Magazine, IEEE, vol. 50, no. 3, pp. 108-115, 2008.

[13] G. Charvat, "A Low-Power Radar Imaging System," in Ph.D. dissertation, Department of Electrical and Computer Engineering, Michigan State University, East Lansing, MI, 2007. 
[14] Y. Wang and G. Zhou, "On the use of high order ambiguity functions for multicomponent polynomial phase signals," Signal Processing, vol. 65, pp. 283-296, 1998.

[15] A. Anghel, G. Vasile, R. Cacoveanu, C. Ioana, and S. Ciochina, "FMCW Transceiver Wideband Sweep Nonlinearity Software Correction," in Proc. IEEE RadarCon2013, Ottawa, Ontario, Canada, May 2013.

[16] J. Yang, J. Thompson, X. Huang, T. Jin, and Z. Zhou, "Randomfrequency sar imaging based on compressed sensing," Geoscience and Remote Sensing, IEEE Transactions on, vol. 51, no. 2, pp. 983-994, 2013.

[17] A. Meta, "Signal processing of FMCW synthetic aperture radar data," in Ph.D. dissertation, Delft Univ. Technol., Delft, The Netherlands, 2006.

[18] United States Department of Defense, "General specification for crystal controlled oscillator. Specification MIL-PRF-55310," Columbus, OH.

[19] P. Brennan, Y. Huang, M.Ash, and K. Chetty, "Determination of sweep linearity requirements in FMCW radar systems based on simple voltagecontrolled oscillator sources," IEEE Trans. Aerosp. Electron. Syst., vol. 47, no. 3, pp. 1594-1604, Jul. 2011.

[20] W. G. Carrara, R. S. Goodman, and R. M. Majewski, Spotlight Synthetic Aperture Radar: Signal Processing Algorithms. Boston: Artech House, 1995.

[21] S. Barbarossa, A. Scaglione, and G. B. Giannakis, "Product highorder ambiguity function for multicomponent polynomial-phase signal modeling," IEEE Trans. Signal Processing, vol. 46, no. 3, pp. 691-708, Mar. 1998.

[22] B. Porat and B. Friedlander, "Accuracy analysis of estimation algorithms for parameters of multiple polynomial-phase signals," in Proc. IEEE Int. Conf. Acoust., Speech, Signal Processing, Detroit, MI, May 1995, pp. $1800-1803$.

[23] S. Peleg and B. Porat, "Estimation and classification of signals with polynomial phase," IEEE Trans. Inform. Theory, vol. 37, no. 2, pp. 422430, 1991.

[24] C. Ioana and A. Quinquis, "Time-frequency analysis using warpedbased high-order phase modeling," EURASIP Journal on Applied Signal Processing, vol. 17, pp. 2856-2873, 2005.

[25] F. A. Marvasti, Nonuniform Sampling:Theory and Practice. New York: Kluwer Academic/Plenum Publishers, 2001.

[26] E. Avignon-Meseldzija, W. Liu, H. Feng, S. Azarian, M. Lesturgie, and Y. Lu, "Compensation of analog imperfections in a Ka-band FMCW SAR," in Proc. EuSAR' 12, Nuremberg, Germany, Apr. 2012, pp. 60-63.

[27] M. Soumekh, Synthetic Aperture Radar Signal Processing with MATLAB Algorithms. New York: Wiley-Interscience, 1999.

[28] R. Touzi, A. Lopes, J. Bruniquel, and P. Vachon, "Coherence estimation for SAR imagery," Geoscience and Remote Sensing, IEEE Transactions on, vol. 37, no. 1, pp. 135-149, 1999.

[29] A. Anghel, G. Vasile, J.-P. Ovarlez, G. d'Urso, and D. Boldo, "Stable scatterers detection and tracking in heterogeneous clutter by repeat-pass SAR interferometry," in Proc. EuSAR'12, Nuremberg, Germany, Apr 2012, pp. 477-480.

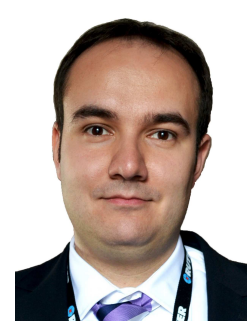

Andrei Anghel (S'11) received the Engineer's degree (as valedictorian) and the Master's degree (with the highest grade) in electronic engineering and telecommunications from the University POLITEHNICA of Bucharest, Bucharest, Romania, in 2010 and 2012, respectively. He is currently working for a joint Ph.D. degree in the field of radar signal processing from the Grenoble Institute of Technology, Grenoble, France and the University POLITEHNICA of Bucharest, Romania. Since 2012 he is Teaching Assistant at the Faculty of Electronics, Telecommunications and Information Technology within the University POLITEHNICA of Bucharest. Between 2010-2011 he has worked in the field of metamaterial composite right/left-handed (CRLH) antennas. In 2012 he pursued a research internship at the Grenoble Image sPeech Signal Automatics Laboratory (GIPSA-Lab), Grenoble, France, on ground-based FMCW radar signal processing. His current research interests include microwaves, radar and signal processing with applications in infrastructure monitoring. Mr. Anghel regularly acts as a Reviewer for the IET Electronics Letters and the Progress in Electromagnetics Research (PIER) Journals. He received two gold medals (in 2005 and 2006) at the International Physics Olympiads.

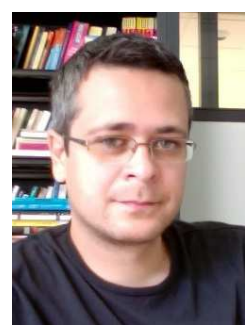

Gabriel Vasile (S'06 - M'07) received the M.Eng. degree in electrical engineering and computer science and the M.S. degree in image, shapes, and artificial intelligence from the POLITEHNICA University, Bucharest, Romania, in 2003 and 2004 respectively, and the Ph.D. degree in signal and image processing from the Savoie University, Annecy, France, in 2007. From 2007 to 2008, he was a Postdoctoral Fellow with the French Space Agency (CNES) and was with the French Aerospace Laboratory (ONERA), Palaiseau, France. In 2008, he joined the French National Council for Scientific Research (CNRS), where he is currently a Research Scientist and a member of the Grenoble Image Speech Signal Automatics Laboratory, Grenoble, France. His research interests include signal and image processing, synthetic aperture radar remote sensing, polarimetry, and interferometry.

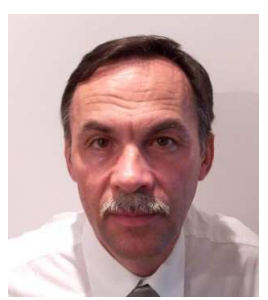

Remus Cacoveanu was born in Romania in 1958 $\mathrm{He}$ received the Master of Science degree in electronics and telecommunications from the POLITEHNICA University of Bucharest, Bucharest, Romania, in 1983, and the Ph.D. degree in optics, optoelectronics and microwave from the Institut $\mathrm{Na}-$ tional Polytechnique de Grenoble, Grenoble, France, in 1997. In 1983, he joined the POLITEHNICA University of Bucharest as a research engineer. $\mathrm{He}$ is currently an Associate Professor with the Department of Telecommunications at the POLITEHNICA University of Bucharest. He worked with the Redline Communications Inc. (2000-2009). Since 2011, he is with the Binq Networks Inc, Canada.

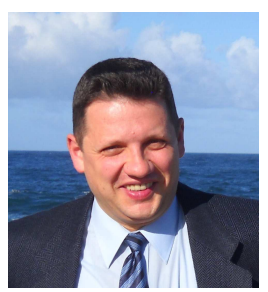

Cornel Ioana received the Dipl.-Eng. degree in electrical engineering from the Romanian Military Technical Academy of Bucharest, Bucharest, Romania, in 1999 and the M.S. degree in telecommunication science and the Ph.D. degree in the electrical engineering field, both from University of Brest, Brest, France, in 2001 and 2003, respectively. Between 1999 and 2001, he activated as a Military Researcher in a research institute of the Romanian Ministry of Defense (METRA), Bucharest, Romania). Between 2003 and 2006, he worked as Researcher and Development Engineer in ENSIETA, Brest, France. Since 2006, he is Associate Professor?Researcher with the Grenoble Institute of Technology/GIPSA-lab, Grenoble, France. His current research activity deals with the signal processing methods adapted to the natural phenomena. His scientific interests are nonstationary signal processing, natural process characterization, underwater systems, electronic warfare, and real-time systems. 


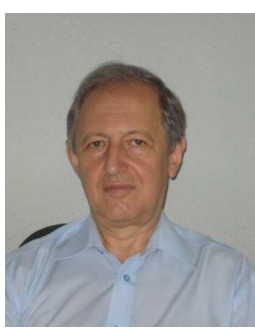

Silviu Ciochina is a professor of the University POLITEHNICA of Bucharest, Bucharest, Romania, and head of the Telecommunications Department, Faculty of Electronics, Telecommunications and Information Technology (since 2003). His main areas of interest are digital signal processing, adaptive algorithms, wireless communication technologies. $\mathrm{He}$ is author of more than 100 papers published in international journals and conference proceedings, seven of them being distinguished with a paper award. Prof. Ciochina is author or coauthor of 10 books, three of them published on international book companies. He was awarded by the Romanian Academy (Traian Vuia-1981 and Gheorghe Cartianu-1999 prizes), the Education Ministry and Defense Ministry (for works in the Radar field). He has been involved in many national and international R\&D projects in the areas of communications, signal processing and radar. $\mathrm{He}$ was the coordinator of the Romanian teams implied in the FP6 projects ATHENA (STREP- 507312), ENTHRONE 1 (IP-507637), and ENTHRONE 2 (IP 038463). 